\title{
Rainbow Turán Problems
}

\author{
PETER KEEVASH, ${ }^{1}$ DHRUV MUBAYI, ${ }^{2 \dagger}$ \\ BENNY SUDAKOV $3 \$$ and JACQUES VERSTRAËTE \\ ${ }^{1}$ Department of Mathematics, Caltech, Pasadena, CA 91125, USA \\ (e-mail: keevash@caltech.edu) \\ ${ }^{2}$ Department of Mathematics, Statistics and Computer Science, University of Illinois, Chicago, IL 60607 \\ (e-mail: mubayi@math.uic.edu) \\ ${ }^{3}$ Department of Mathematics, Princeton University, Princeton, NJ 08544 \\ (e-mail: bsudakov@math.princeton.edu) \\ ${ }^{4}$ Department of Combinatorics and Optimization, Faculty of Mathematics, University of Waterloo, \\ Waterloo, Ontario, Canada, N2V2K7 \\ (e-mail: jverstraete@math.uwaterloo.ca)
}

Received 7 June 2004; revised 7 October 2005

\begin{abstract}
For a fixed graph $H$, we define the rainbow Turán number $\operatorname{ex}^{*}(n, H)$ to be the maximum number of edges in a graph on $n$ vertices that has a proper edge-colouring with no rainbow $H$. Recall that the (ordinary) Turán number ex $(n, H)$ is the maximum number of edges in a graph on $n$ vertices that does not contain a copy of $H$. For any non-bipartite $H$ we show that $\operatorname{ex}^{*}(n, H)=(1+o(1)) \operatorname{ex}(n, H)$, and if $H$ is colour-critical we show that $\operatorname{ex}^{*}(n, H)=\operatorname{ex}(n, H)$. When $H$ is the complete bipartite graph $K_{s, t}$ with $s \leqslant t$ we show $\operatorname{ex}^{*}\left(n, K_{s, t}\right)=O\left(n^{2-1 / s}\right)$, which matches the known bounds for ex $\left(n, K_{s, t}\right)$ up to a constant. We also study the rainbow Turán problem for even cycles, and in particular prove the bound $\operatorname{ex}^{*}\left(n, C_{6}\right)=O\left(n^{4 / 3}\right)$, which is of the correct order of magnitude.
\end{abstract}

\section{Introduction}

In this paper, we address the following question. For a fixed graph $H$, determine the maximum number of edges in a properly edge-coloured graph on $n$ vertices which does not contain a rainbow $H$, i.e., a copy of $H$, all of whose edges have different colours. This maximum is denoted $\operatorname{ex}^{*}(n, H)$, and we refer to it as the rainbow Turán number of $H$.

$\dagger$ Research supported in part by NSF grant DMS-0400812, and by an Alfred P. Sloan fellowship.

$\ddagger$ Research supported in part by NSF grant DMS-0355497, a USA-Israeli BSF grant, and by an Alfred P. Sloan fellowship. 
There are two main motivations for our study of rainbow Turán numbers. One is the possibility of applying purely combinatorial methods to certain extremal problems in additive number theory. Call a subset $A$ of an abelian group $G$ a $B_{k}^{*}$-set if does not contain disjoint $k$-subsets $B, C$ with the same sum. Given a set $A$, consider the following edge-coloured bipartite graph. The two parts $X, Y$ are both copies of $G$; we join $x \in X$ to $y \in Y$ if $x-y \in A$, and then the edge $x y$ is assigned the colour $x-y$. This is a properly coloured graph, and if $A$ is a $B_{k}^{*}$-set then it does not contain a rainbow $C_{2 k}$, the cycle of length $2 k$. A similar approach, involving properly coloured bipartite graphs, was taken by Ruzsa and Szemerédi [29] to give a purely combinatorial proof of Roth's theorem [27] on three-term arithmetic progressions.

Another motivation is that it seems to be a natural meeting point of two areas of extremal graph theory. Firstly, there is the classical Turán problem, which has a rich history in combinatorics. This asks for the maximum number of edges in a graph on $n$ vertices that contains no copy of some fixed graph $H$. The maximum here is denoted ex $(n, H)$, and is known as the Turán number for $H$. Next there is the literature on extremal problems for edge-colourings (not necessarily proper). An example is the canonical Ramsey theorem, proved by Erdős and Rado [12], a special case of which shows that any proper colouring of $K_{n}$ produces a rainbow $K_{m}$, provided $n$ is large relative to $m$. Motivated by this and work in [10] and [19], Alon, Jiang, Miller and Pritikin [2] introduced the problem of finding a rainbow copy of a graph $H$ in a colouring of $K_{n}$ in which each colour appears at most $m$ times at each vertex. The rainbow Turán problem is a natural Turán-type extension of this problem.

We will discuss these motivations in greater detail before the statement of our relevant results, which we divide into subsections according to the nature of the forbidden fixed graph $H$.

\subsection{Preliminary results}

For the sake of completeness, we start by presenting some results that can be easily deduced from the corresponding results for the ordinary Turán problem. For the reader's convenience we first give some background information on this (ordinary) problem. Its systematic study originated with Turán, who considered forbidding $K_{r}$, the complete graph on $r$ vertices. The Turán graph $T_{r-1}(n)$ is the complete $(r-1)$-partite graph with part sizes as equal as possible; we write $t_{r-1}(n)$ for the number of edges in $T_{r-1}(n)$. Then Turán's theorem [32] states that $\operatorname{ex}\left(n, K_{r}\right)=t_{r-1}(n)$, and $T_{r-1}(n)$ is the unique extremal $K_{r}$-free graph. Erdős and Stone [14] showed that the behaviour of the Turán number of a general graph $H$ is determined by its chromatic number. They proved that if $\chi(H)=r$, then $\operatorname{ex}(n, H)=t_{r-1}(n)+o\left(n^{2}\right)$, which gives asymptotics except when $H$ is bipartite.

Clearly the rainbow Turán number for any $H$ satisfies $\operatorname{ex}^{*}(n, H) \geqslant \operatorname{ex}(n, H)$. Examples when equality holds include the cases when $H$ is a star or a triangle, as then any proper edge-colouring of $H$ is rainbow, and so $\operatorname{ex}^{*}(n, H)=\operatorname{ex}(n, H)$. We can describe a general class of graphs in which equality holds as follows. We say that $H$ is colour-critical if it contains an edge $e$ so that $\chi(H \backslash e)=\chi(H)-1$, where $\chi(H)$ denotes the chromatic 
number of $H .{ }^{1}$ If $H$ is colour-critical and $\chi(H)=r$, then a result of Simonovits [30] shows that $\operatorname{ex}(n, H)=t_{r-1}(n)$ for sufficiently large $n$. Our first result determines ex $(n, H)$ asymptotically for any non-bipartite $H$ and exactly for colour-critical graphs, a class that includes, for example, all complete graphs and all cycles of odd length.

Proposition 1.1. The rainbow Turán number $\operatorname{ex}^{*}(n, H)$ satisfies $\operatorname{ex}(n, H) \leqslant \operatorname{ex}^{*}(n, H) \leqslant$ $\operatorname{ex}(n, H)+o\left(n^{2}\right)$. Furthermore, if $H$ is colour-critical then $\operatorname{ex}^{*}(n, H)=\operatorname{ex}(n, H)$ for $n$ sufficiently large.

The first statement of Proposition 1.1 can be generalized along the lines of [2]: any edge-coloured graph $G$ on $n$ vertices, with $\operatorname{ex}\left(n, K_{r}\right)+\epsilon n^{2}$ edges, and $o(n)$ edges of the same colour at each vertex, contains a rainbow $K_{r}$. To see this, one first observes that $G$ contains $o\left(n^{r}\right)$ non-rainbow copies of $K_{r}$. On the other hand, it follows from an inequality of Moon and Moser [25] that $G$ contains $\Omega\left(n^{r}\right)$ copies of $K_{r}$, and therefore some $K_{r} \subset G$ must be rainbow. The following construction shows that the second statement of Proposition 1.1 cannot be extended along these lines, even when $H$ is a triangle: take a balanced complete $n$ by $n$ bipartite graph with parts $A$ and $B$ and add a matching to $A$. Colour the edges so that edges $e_{1}$ and $e_{2}$ have the same colour if and only if there is a vertex $b \in B$ and an edge $a_{1} a_{2}$ in $A$ so that $e_{1}=b a_{1}$ and $e_{2}=b a_{2}$. This graph has no rainbow triangles, at most two colours at each vertex, and has ex $\left(2 n, K_{3}\right)+n$ edges.

We will see later that for some bipartite graphs $H$ there is a considerable gap between $\operatorname{ex}(n, H)$ and $\operatorname{ex}^{*}(n, H)$. In general, even the order of magnitude of Turán numbers for bipartite graphs is not well understood. In the case of complete bipartite graphs, Kővári, Sós and Turán [21] showed ex $\left(n, K_{s, t}\right)=O\left(n^{2-1 / s}\right)$, where the implied constant depends only on $s$ and $t$. The best-known bound on the constant is due to Füredi [15]. For $t>(s-1)$ ! there is a lower bound of the same order of magnitude given by a construction of [4] (modifying that of [20]). Generalizing the upper bound for $K_{s, t}$, Alon, Krivelevich and Sudakov [3] showed $\operatorname{ex}(n, H)=O\left(n^{2-1 / s}\right)$ whenever $H$ is a bipartite graph in which the vertices of one part all have degree at most $s$. We note that one can easily deduce the following rainbow version of this result.

Proposition 1.2. Let $H$ be a bipartite graph in which the vertices of one part all have degree at most $s$. Then $\operatorname{ex}^{*}(n, H)=O\left(n^{2-1 / s}\right)$.

It seems difficult to determine whether $\operatorname{ex}^{*}\left(n, K_{s, t}\right) \sim \operatorname{ex}\left(n, K_{s, t}\right)$, even in the simplest case $s=t=2$. This leads us to our next topic, the rainbow Turán problem for even cycles.

\subsection{Even cycles and $B_{k}$-sets}

The case of even cycles is of particular interest, not only in the context of rainbow Turán numbers, but in its relation to the problem of $B_{k}$-sets in combinatorial number theory.

\footnotetext{
${ }^{1}$ Note that our definition is non-standard; often the term means that deleting any edge reduces the chromatic number.
} 
Here our lower bound comes from a construction of Bose and Chowla [9], but to get a matching upper bound we need an extra assumption.

Theorem 1.3. For all $k \geqslant 2$, there exists an absolute constant $c>0$ such that $\operatorname{ex}^{*}\left(n, C_{2 k}\right) \geqslant$ $c n^{1+1 / k}$. Furthermore, if $G$ is a properly edge-coloured graph without any cycles of length less than $2 k$ and without any rainbow cycle of length $2 k$, then $G$ has $O\left(n^{1+1 / k}\right)$ edges.

An upper bound ex $\left(n, C_{2 k}\right)<c(k) n^{1+1 / k}$ was obtained by Bondy and Simonovits [8]. The best-known bound on the constant $c(k)$ is due to Verstraëte [33]. Based on the evidence of Theorem 1.3 we conjecture that $\operatorname{ex}^{*}\left(n, C_{2 k}\right)$ has the same order of magnitude.

Conjecture 1.4. For all $k \geqslant 2$, $\operatorname{ex}^{*}\left(n, C_{2 k}\right)=O\left(n^{1+1 / k}\right)$.

Remarks. (1) It is a little surprising that one can find a lower bound $\operatorname{ex}^{*}\left(n, C_{2 k}\right) \geqslant c n^{1+1 / k}$ when all the known constructions of $C_{2 k}$-free graphs have much fewer edges. Lower bounds on $\operatorname{ex}\left(n, C_{2 k}\right)$ of order $n^{1+1 / k}$ are only known for $k$ equal to 2,3 or 5 .

(2) Rödl and Tuza [26] proved the existence of graphs of arbitrarily large girth for which any proper edge-colouring produces a rainbow cycle. They show that random graphs have this property, but it would be interesting to give explicit examples of such graphs. The second part of Theorem 1.3 would provide this if we could prove the existence of certain conjectured constructions of graphs without short (even) cycles. For example, there are constructions of bipartite graphs with no cycles of length at most 10 with $\Omega\left(n^{6 / 5}\right)$ edges, and our theorem implies that any proper edge-colouring of such a graph produces a rainbow $C_{12}$. The best-known general constructions are given by Lazebnik, Ustimenko and Woldar [22], who construct graphs with no cycle of length less than $2 k$ with $\Omega\left(n^{1+1 / \ell}\right)$ edges, where $\ell$ is approximately $3 k / 2$.

Although we cannot prove Conjecture 1.4 in general, we prove it in the case $k=3$. (The case $k=2$ is covered by Proposition 1.2, which gives the bound $\operatorname{ex}^{*}\left(n, K_{2,2}\right)=O\left(n^{3 / 2}\right)$.)

Theorem 1.5. There exist absolute constants $c_{2} \geqslant c_{1}>1$ such that

$$
c_{1} \operatorname{ex}\left(n, C_{6}\right) \leqslant \operatorname{ex}^{*}\left(n, C_{6}\right) \leqslant c_{2} \operatorname{ex}\left(n, C_{6}\right) .
$$

In particular, $\operatorname{ex}^{*}\left(n, C_{6}\right)=\Theta\left(n^{4 / 3}\right)$.

Note that this theorem demonstrates that $\operatorname{ex}^{*}\left(n, C_{6}\right)$ is not asymptotically equal to $\operatorname{ex}\left(n, C_{6}\right)$, in contrast to the non-bipartite case.

A $B_{k}^{*}$-set in an abelian group $G$ is a set $A \subset G$ with the property that no pair of disjoint $k$-element subsets of $A$ have the same sum. Later we will describe a simple construction, for which applying Theorem 1.5 implies $|A|=O\left(|G|^{1 / 3}\right)$. This gives the correct order of magnitude for the maximum size of a $B_{3}^{*}$-set, although the constant that we obtain is weaker than that of previously known bounds. The construction also gives an extra motivation for considering Conjecture 1.4, as it could potentially give a purely 
combinatorial way to determine the correct order of magnitude for the maximum size of a general $B_{k}^{*}$-set. We will give a more detailed discussion of this connection in Section 3 .

\subsection{Excluding all cycles}

A graph on $n$ vertices without any cycle at all has at most $n-1$ edges, but how many edges can there be in a properly coloured graph without a rainbow cycle? By contrast with the ordinary Turán problem, we can give a construction with $\Omega(n \log n)$ edges. On the other hand, we cannot improve the upper bound of $O\left(n^{4 / 3}\right)$ given by Theorem 1.5.

Proposition 1.6. For any $m$ there is a graph with $6^{m}$ vertices and $6^{m} \cdot 3 m / 2$ edges that can be properly coloured with no rainbow cycle.

In fact we can construct graphs with $\Omega(n \log n)$ edges with no cycle that uses more than half as many colours as edges, so it is natural to relax our problem and ask how many edges are sufficient to find such a cycle. An additional motivation is that it implies a bound for graphs in which each cycle uses all of its colours at least twice; these are non-rainbow in a particularly strong way.

Theorem 1.7. Let $G$ be a graph on $n$ vertices so that for all $k$, any cycle of length $2 k$ uses at most $k$ different colours. Then the number of edges of $G$ satisfies $e(G)<n \log _{2}(n+3)-2 n$. Furthermore, when $n$ is a power of 2 then there is an example of such a graph with $\frac{1}{2} n \log _{2} n$ edges.

The rest of this paper is organized as follows. In the next section we prove Propositions 1.1 and 1.2 by reductions to appropriate ordinary Turán problems. Section 3 contains the proofs of the theorems on cycles and $B_{k}^{*}$-sets. In the last section of the paper we have some concluding remarks and open problems.

\section{Reductions to Turán problems}

In this section we show how, for certain graphs $H$, the rainbow Turán problem for a graph $H$ can be reduced to the ordinary Turán problem for some larger graph $H^{\prime}$. We will use this method to prove Propositions 1.1 and 1.2. The reductions are based on a simple greedy algorithm, which we formulate as follows.

Lemma 2.1. Suppose $G$ is a properly edge-coloured graph and $X$ is a subset of its vertices for which the induced graph $G_{X}$ is rainbow. If $Y$ is any set of vertices disjoint from $X$ with $|Y|>(|X|-2) e\left(G_{X}\right)$ then there is a vertex $y \in Y$ so that $X \cup\{y\}$ induces a rainbow subgraph of $G$.

Proof. Let $C$ be the set of colours that appear on the edges of $G_{X}$. By assumption $|C|=e\left(G_{X}\right)$. For each $x \in X$ let $d_{x}$ denote the degree of $x$ in $G_{X}$. There are at most $|C|$ vertices $y$ such that $x y$ has a colour in $C$, so at most $|C|-d_{x}$ such vertices in $Y$. Therefore the number of vertices in $Y$ that are joined to any vertex in $X$ by an edge with a colour 
in $C$ is at most $\sum_{x \in X}\left(|C|-d_{x}\right)=|X||C|-2 e\left(G_{X}\right)=(|X|-2) e\left(G_{X}\right)$. Since $|Y|$ is larger than this we can choose $y \in Y$ so that no colour in $C$ appears on the edges from $y$ to $X$. Since $G$ is properly edge-coloured, these edges all have different colours, so $X \cup\{y\}$ induces a rainbow subgraph.

From this we deduce the following lemma, which provides the reduction for Proposition 1.1. First we need some notation. We write $K_{r}(t)$ for the complete $r$-partite graph with $t$ vertices in each class, and $K_{r}(t)^{+}$for the graph obtained from $K_{r}(t)$ by adding an edge to one of the classes.

\section{Lemma 2.2.}

(1) Any proper colouring of $K_{r}\left(r^{3} t^{3}\right)$ contains a rainbow $K_{r}(t)$ and

(2) any proper colouring of $K_{r}\left(r^{3} t^{3}\right)^{+}$contains a rainbow $K_{r}(t)^{+}$.

Proof. It suffices to prove the second statement. For then, given any properly coloured $K_{r}\left(r^{3} t^{3}\right)$, we can add an edge inside a class with some new colour and find a rainbow $K_{r}(t)^{+}$. This must use the added edge, which we delete to get a rainbow $K_{r}(t)$ in the original graph. Consider then a properly coloured $K_{r}\left(r^{3} t^{3}\right)^{+}$with parts $X_{1}, \ldots, X_{r}$ in which the extra edge joins $a$ and $b$ in $X_{1}$. We need to find $Y_{1}, \ldots, Y_{r}$ spanning a rainbow subgraph, where $Y_{i} \subset X_{i}$ has size $t$ for each $i$ and $\{a, b\} \subset Y_{1}$. This can be achieved by selecting each $Y_{i}$ in turn by the greedy algorithm, starting with $Y_{1}$ which can be any $t$ vertices of $X_{1}$ including $a$ and $b$. At any stage we have selected at most $t r$ vertices and they span at most $t^{2}\left(\begin{array}{l}r \\ 2\end{array}\right)$ edges. We need to choose the next vertex to belong to some $X_{i}$ and not be one of the previously chosen vertices, so that the subgraph spanned is again rainbow. This is possible by Lemma 2.1 as $\left|X_{i}\right|=r^{3} t^{3}>t r+(t r-2) t^{2}\left(\begin{array}{c}r \\ 2\end{array}\right)$.

Proof of Proposition 1.1. Suppose $H$ is a graph on $t$ vertices with chromatic number $r$. Then $H$ is a subgraph of $K_{r}(t)$. By the Erdős-Stone theorem [14], any graph $G$ on $n$ vertices with $\operatorname{ex}\left(n, K_{r}\right)+o\left(n^{2}\right)$ edges will contain a copy of $K_{r}\left(r^{3} t^{3}\right)$. Then a proper colouring of $G$ will yield a rainbow $K_{r}(t)$ by Lemma 2.2, which contains a rainbow $H$. Therefore $\operatorname{ex}^{*}(n, H)<\operatorname{ex}\left(n, K_{r}\right)+o\left(n^{2}\right)=\operatorname{ex}(n, H)+o\left(n^{2}\right)$.

Now suppose, in addition, that $H$ is colour-critical. Then, $H$ is a subgraph of $K_{r-1}(t)^{+}$. Note that $K_{r-1}\left((r-1)^{3} t^{3}\right)^{+}$is colour-critical. Then, by the result of Simonovits mentioned earlier, any graph $G$ on $n$ vertices with more than $\operatorname{ex}\left(n, K_{r}\right)$ edges will contain a copy of $K_{r-1}\left((r-1)^{3} t^{3}\right)^{+}$, for $n$ sufficiently large. A proper colouring of $G$ will yield a rainbow $K_{r-1}(t)^{+}$by Lemma 2.2, which contains a rainbow $H$. Therefore $\operatorname{ex}^{*}(n, H)=\operatorname{ex}(n, H)=$ $\operatorname{ex}\left(n, K_{r}\right)$.

Example. We remark that $\operatorname{ex}^{*}(n, H)$ is not equal to $\operatorname{ex}(n, H)$ for a general non-bipartite graph. Consider, for example, a graph $H$ that consists of $t$ triangles that all share exactly one common vertex (a ' $t$-fan'). Erdős, Füredi, Gould and Gunderson [11] showed that $\operatorname{ex}(n, H)$ is equal to $\left\lfloor n^{2} / 4\right\rfloor+t^{2}-t$ for $t$ odd and $\left\lfloor n^{2} / 4\right\rfloor+t^{2}-3 t / 2$ for $t$ even, when $n \geqslant 50 t^{2}$. On the other hand, we have $\operatorname{ex}^{*}(n, H) \geqslant\left\lfloor n^{2} / 4\right\rfloor+(t-1)\lfloor n / 2\rfloor$, as shown by the following construction. Start with any proper colouring of the Turán graph $T_{2}(n)$. 
Now add $t-1$ new matchings to the graph, each with its own new colour. Any rainbow subgraph of this construction uses at most $t-1$ edges that did not come from the Turán graph. On the other hand it is impossible to obtain a bipartite graph by deleting $t-1$ edges from a $t$-fan, so the construction does not have a rainbow $t$-fan. Therefore for any constant $C$ there is a non-bipartite graph $H$ with $\operatorname{ex}^{*}(n, H)-\operatorname{ex}(n, H)>C n$ for large $n$.

Next we prove Proposition 1.2, which states that if $H=(X, Y)$ is any bipartite graph in which the vertices of $X$ all have degree at most $s$, then $\operatorname{ex}^{*}(n, H)=O\left(n^{2-1 / s}\right)$. Given a graph $G$, call a subset $A$ of vertices $(s, b)$-common if every $s$ vertices in $A$ have at least $b$ common neighbours. We use the following consequence of Lemma 2.1 of Alon, Krivelevich and Sudakov [3]: for any $a, b, s$ there is a constant $c$ such that any graph on $n$ vertices with at least $c n^{2-1 / s}$ edges contains an $(s, b)$-common set of size $a$. When $H$ has $h$ vertices we choose $a=h$ and $b=h^{3}$.

Proof of Proposition 1.2. Let $H=(X, Y)$ and $h, c$ be as defined in the previous paragraph. Suppose $G$ is a properly coloured graph on $n$ vertices with at least $c n^{2-1 / s}$ edges. By definition there is an $\left(s, h^{3}\right)$-common set $A$ of size $h$. Choose any set $Y^{\prime}$ of $|Y|$ vertices in $A$ to represent the part $Y$ of $H$. We select vertices of $G$ to represent $X$ by a greedy algorithm. Suppose we have come to some $x$ in $X$. Let $Y_{x} \subset Y$ be its neighbours in $H$ and let $Y_{x}^{\prime}$ be their representatives in $Y^{\prime}$. We have already chosen fewer than $h$ vertices, so there are fewer than $\left(\begin{array}{l}h \\ 2\end{array}\right)$ forbidden colours, and so fewer than $h\left(\begin{array}{l}h \\ 2\end{array}\right)<h^{3}$ vertices that cannot be used as a representative for $x$. By definition $\left|Y_{x}^{\prime}\right| \leqslant s$, so there are at least $h^{3}$ common neighbours of $Y_{x}^{\prime}$ in $G$. We can choose any of these that is not forbidden as a representative of $x$.

There are certain complete bipartite graphs for which the Turán numbers are known asymptotically, not just to order of magnitude. Füredi showed in [15] that ex $\left(n, K_{3,3}\right)=$ $(1+o(1)) \frac{1}{2} n^{5 / 3}$ and in [16] that $\operatorname{ex}\left(n, K_{2, t}\right)=(1+o(1)) \frac{1}{2} \sqrt{t-1} n^{3 / 2}$. In the following lemma we analyse our greedy procedure more carefully to get the best constants achievable by our reduction method.

Lemma 2.3. Suppose $s, t>1$ and $t^{\prime}>(s(s-1)+1)(t-1)$. Then any proper colouring of $K_{s, t^{\prime}}$ contains a rainbow $K_{s, t}$.

Proof. Let $K_{s, t^{\prime}}$ be properly coloured, and denote the parts by $A, B$ where $|A|=s,|B|=t^{\prime}$. Suppose we have chosen $v_{1}, \ldots, v_{p}$ in $B$ so that $A \cup\left\{v_{1}, \ldots, v_{p}\right\}$ spans a rainbow subgraph $H$, for some $p<t$. Let $C$ be the colours appearing on edges of $H$, so that $|C|=p$ s. For each $a$ in $A$ there are at most $p(s-1)$ vertices $b$ in $B \backslash\left\{v_{1}, \ldots, v_{p}\right\}$ for which $a b$ has a colour in $C$. Then there are at most $p+|A| \cdot p(s-1)=p(1+s(s-1))<t^{\prime}$ unavailable vertices in $B$, so we can choose $v_{p+1}$.

Remark. We can give a construction that shows that the bound for $t^{\prime}$ in Lemma 2.3 cannot be improved in general, which suggests that an improvement in the constant for $\operatorname{ex}^{*}\left(n, K_{s, t}\right)$ will not come from a reduction to an ordinary Turán problem. It is based on 
the well-known result of Singer [31] that when $q$ is a prime power there is a $(v, q+1,1)$ difference set $D$ in $\mathbb{Z} / v \mathbb{Z}$, where $v=q^{2}+q+1$. (For definitions and a proof see [23, Chapter 27].) Our construction is to take a complete bipartite graph with parts $D$ and $\mathbb{Z} / v \mathbb{Z} \times\{1, \ldots, t-1\}$, where for $d \in D, x \in \mathbb{Z} / v \mathbb{Z}$ and $1 \leqslant i \leqslant t-1$ we colour the edge joining $d$ to $(x, i)$ with the pair $(d+x, i)$. We leave the interested reader to verify that when $s=q+1$ we have a proper colouring of $K_{s,(s(s-1)+1)(t-1)}$ with no rainbow $K_{s, t}$.

It follows from this lemma, and the result of Füredi previously mentioned, that $\operatorname{ex}^{*}\left(n, K_{2, t}\right)<(1+o(1)) \frac{1}{2} \sqrt{3(t-1)} n^{3 / 2}$. It is natural to ask whether this constant may be improved. For instance, in the simplest case of quadrilaterals $\left(C_{4}=K_{2,2}\right)$ we only know that the inferior and superior limits of $\operatorname{ex}^{*}\left(n, C_{4}\right) / n^{3 / 2}$ lie between $1 / 2$ and $\sqrt{3} / 2$.

Problem 2.4. Determine the asymptotic behaviour of $\operatorname{ex}^{*}\left(n, C_{4}\right)$ : that is, prove that the limit

$$
\lim _{n \rightarrow \infty} \frac{\operatorname{ex}^{*}\left(n, C_{4}\right)}{n^{3 / 2}}
$$

exists and determine its value.

Example. For bipartite graphs $H$, it is not necessarily the case that $\operatorname{ex}^{*}(n, H)$ is asymptotically equal to $\operatorname{ex}(n, H)$. The path with $t$ edges $P_{t}$ is a counterexample whenever $t$ is of the form $2^{k}-1$ for an integer $k \geqslant 2$. It is well known and easy to show that $\operatorname{ex}\left(n, P_{t}\right) \leqslant(t-1) n / 2$. (Equality can hold for a graph that is a disjoint union of copies of $K_{t}$.) On the other hand, Maamoun and Meyniel [24] give an example of a proper colouring of $K_{2^{k}}$ containing no rainbow path with $2^{k}-1$ edges. (The vertices are identified with the vectors $\mathbb{F}_{2}^{k}$ and an edge is coloured by the difference of its vertices.) Taking a disjoint union of such $K_{2^{k}}$ S we obtain $\operatorname{ex}^{*}\left(n, P_{2^{k}-1}\right) \geqslant\left(\begin{array}{c}2^{k} \\ 2\end{array}\right)\left\lfloor n / 2^{k}\right\rfloor=(1+o(1)) \frac{2^{k}-1}{2^{k}-2} \operatorname{ex}\left(n, P_{2^{k}-1}\right)$.

In general, it seems an interesting problem to determine the asymptotics of $\operatorname{ex}^{*}\left(n, P_{t}\right)$. We remark that it is not hard to show that for the simplest case $P_{3}$ the above construction is essentially tight, in that $\operatorname{ex}^{*}\left(n, P_{3}\right) \leqslant 3 n / 2$. (The key observation is that if $G$ has no rainbow $P_{3}$ and $d(x) \geqslant 4$ then there are no edges incident to the neighbours of $x$ other than those incident to $x$.) A natural conjecture is that the optimal construction should be a disjoint union of cliques of size $c(t)$, where $c(t)$ is chosen as large as possible so that the cliques can be properly coloured with no rainbow $P_{t}$. It is not hard to see that $t \leqslant c(t) \leqslant 2(t-1)$, where the upper bound is our usual greedy argument. Even the problem of determining $c(t)$ exactly may be difficult.

Problem 2.5. Determine the asymptotic behaviour of $\operatorname{ex}^{*}\left(n, P_{t}\right)$.

\section{Even cycles and $B_{k}^{*}$-sets}

For a subset $A$ of an abelian group $G$, we define the coloured bipartite Cayley graph as follows. The two parts $X, Y$ are both copies of $G$, we join $x \in X$ to $y \in Y$ if $y-x \in A$, and then the edge $x y$ is assigned the colour $y-x$. Note that this is a properly coloured graph. 
Suppose that $x_{1} y_{1} \cdots x_{k} y_{k}$ is a rainbow cycle of length $2 k$. Let $B=\left\{y_{1}-x_{1}, \ldots, y_{k}-x_{k}\right\}$ and $C=\left\{y_{1}-x_{2}, \ldots, y_{k-1}-x_{k}, y_{k}-x_{1}\right\}$. Then $B, C$ are disjoint $k$-subsets of $A$ with the same sum. We say $A$ is a $B_{k}^{*}$-set if no such subsets exist. Thus a bound on the number of edges in a graph with no rainbow $C_{2 k}$ gives a bound on the size of a $B_{k}^{*}$-set.

A related and more commonly studied condition is the following. We call $A$ a $B_{k}$-set if any element $g \in G$ has at most one representation of the form $g=a_{1}+\cdots+a_{k}$ with $a_{i} \in A$ for $1 \leqslant i \leqslant k$, where we do not count permutations of the summands as being a different representation. There are $\left(\begin{array}{c}|A|+k-1 \\ k\end{array}\right)$ different representations, so if $|G|=n$ we have $|A|<(k ! n)^{1 / k}$. When $G=\mathbb{Z} / n \mathbb{Z}$ Bose and Chowla [9] constructed $B_{k}$ sets of size $(1+o(1)) n^{1 / k}$, showing that $n^{1 / k}$ is the correct order of magnitude. Note that a $B_{k}$-set is, in particular, a $B_{k}^{*}$-set, so there are $B_{k}^{*}$-sets in $\mathbb{Z} / n \mathbb{Z}$ of size $(1+o(1)) n^{1 / k}$. An upper bound of the same order of magnitude was obtained by Ruzsa [28], who showed that a $B_{k}^{*}$-set in the integers $\{1, \ldots, n\}$ has at most $(1+o(1)) k^{2-1 / k} n^{1 / k}$ elements. One of the outstanding problems in combinatorial number theory is to close the gap between the upper and lower bounds for such sets.

\subsection{Rainbow Turán for even cycles}

In this subsection we prove Theorem 1.3, which concerns the rainbow Turán number $\operatorname{ex}^{*}\left(n, C_{2 k}\right)$. The lower bound $(1+o(1))(n / 2)^{1+1 / k}$ follows from the bipartite Cayley graph construction described above, applied to a Bose-Chowla $B_{k}$-set in $\mathbb{Z} / n \mathbb{Z}$. Now we will show a corresponding upper bound under the additional assumption of there being no strictly shorter cycles in the underlying graph.

Proof of Theorem 1.3. Let $G$ be a graph on $n$ vertices with no cycle of length less than $2 k$. Suppose $G$ has a proper colouring with no rainbow $C_{2 k}$. Let $d=2 e(G) / n$ be the average degree. We will show that $d<2\left(k^{2} n\right)^{1 / k}+4 k$ for large $n$. Note that we can assume that $G$ has minimum degree at least $d / 2$, as deleting a vertex of degree less than $d / 2$ does not decrease the average degree. We start by showing that the number of rainbow paths of length $k$ satisfies

$$
R_{k} \geqslant 2^{-k+1} n d(d-1) \prod_{i=1}^{k-2}(d-4 i) .
$$

This follows by induction on $k$. First of all, by Cauchy-Schwarz there are at least $n\left(\begin{array}{l}d \\ 2\end{array}\right)$ (rainbow) paths of length 2. For $t \geqslant 2$ each rainbow path of length $t+1$ contains 2 rainbow paths of length $t$. Also, given a rainbow path of length $t$, each of its endpoints is incident to at least $d / 2$ edges, of which at most $t-1$ have endpoints on the path and at most $t-1$ others have a colour that appears on the path, so it can be extended to a rainbow path of length $t+1$ in at least $2(d / 2-2(t-1))$ ways. Therefore $R_{t+1} \geqslant(d / 2-2(t-1)) R_{t}$, which proves the claim.

Given a pair of vertices $a, b$, let $p_{a b}$ denote the number of rainbow paths of length $k$ with endpoints $a$ and $b$. Since $G$ has girth at least $2 k$ any two such paths $a x_{1} \cdots x_{k-1} b$ and $a x_{1}^{\prime} \cdots x_{k-1}^{\prime} b$ are internally disjoint, i.e., $a x_{1} \cdots x_{k-1} b x_{k-1}^{\prime} \cdots x_{1}^{\prime}$ is a $2 k$-cycle. By assumption, there are two edges of the same colour on this cycle. Say that a path $y_{1} \cdots y_{t}$ is special 
if there is some $i>1$ such that $y_{1} y_{2}$ and $y_{i} y_{i+1}$ have the same colour, and no other pair of edges have the same colour. We claim that the cycle $a x_{1} \cdots x_{k-1} b x_{k-1}^{\prime} \cdots x_{1}^{\prime}$ contains a special path of length $k+1$.

To see this, we start with the shortest path that contains two edges with the same colour. One of each must appear on the two rainbow paths joining $a$ and $b$, so suppose the path is $x_{i} \cdots x_{1} a x_{1}^{\prime} \cdots x_{j}^{\prime}$ for some $i, j$. Here $x_{i-1} x_{i}$ and $x_{j-1}^{\prime} x_{j}^{\prime}$ have the same colour (using the shorthand $x_{0}=x_{0}^{\prime}=a$ ) and no other pair of edges have the same colour. The length of the path is $i+j$. Note that $x_{i-1} x_{i}$ and $x_{j-1}^{\prime} x_{j}^{\prime}$ belong to the path $x_{i-1} \cdots x_{k-1} b x_{k-1}^{\prime} \cdots x_{j-1}^{\prime}$ of length $2(k+1)-(i+j)$, and as we chose the shortest path it has length $i+j \leqslant k+1$. Now consider the path $x_{t} \cdots x_{1} a x_{1}^{\prime} \cdots x_{k+1-t}^{\prime}$ where $t \geqslant 1$ is chosen as small as possible so that the colour of $x_{t-1} x_{t}$ is repeated on the path. This path exists by the preceding discussion, and there are no other repetitions of colours, as this would contradict the minimality of $t$. Therefore we have found a special path of length $k+1$.

Note that each special path of length $k+1$ contains a rainbow path of length $k$ (obtained by deleting the end-edge whose colour is repeated) and each rainbow path of length $k$ can be extended to at most $2(k-1)$ special paths of length $k+1$. This shows that there are at most $2(k-1) R_{k}$ special paths of length $k+1$. Also, since $G$ has girth at least $2 k$ there is at most one path of length $k-1$ between any two points, so each special path comes from at most one $C_{2 k}$. Each such $C_{2 k}$ can be written as the union of two rainbow paths of length $k$ in at most $k$ ways. We conclude that

$$
\frac{1}{k} \sum_{a, b}\left(\begin{array}{c}
p_{a b} \\
2
\end{array}\right) \leqslant 2(k-1) R_{k}=2(k-1) \sum_{a, b} p_{a b} .
$$

This can be rewritten as $\sum_{a, b} p_{a, b}^{2} \leqslant(4 k(k-1)+1) \sum_{a, b} p_{a b}$. By the Cauchy-Schwarz inequality

$$
\sum_{a, b} p_{a b}^{2} \geqslant\left(\begin{array}{l}
n \\
2
\end{array}\right)^{-1}\left(\sum_{a, b} p_{a b}\right)^{2}
$$

and so we see that $\sum_{a, b} p_{a b} \leqslant(4 k(k-1)+1)\left(\begin{array}{l}n \\ 2\end{array}\right)$. Now by equation (3.1) we have

$$
2^{-k+1} n d(d-1) \prod_{i=1}^{k-2}(d-4 i) \leqslant R_{k}=\sum_{a, b} p_{a b} \leqslant(4 k(k-1)+1)\left(\begin{array}{l}
n \\
2
\end{array}\right),
$$

which implies $d<2\left(k^{2} n\right)^{1 / k}+4 k$.

\subsection{Rainbow Turán for $C_{6}$}

In this subsection we discuss the rainbow Turán problem for the six-cycle (or hexagon). For the ordinary Turán problem the best-known bounds are due to Füredi, Naor and Verstraëte [17]. They show that $(1+o(1)) \alpha n^{4 / 3} \leqslant \operatorname{ex}\left(n, C_{6}\right) \leqslant(1+o(1)) \beta n^{4 / 3}$, where $\alpha=$ $3(\sqrt{5}-2)(\sqrt{5}-1)^{-4 / 3} \sim 0.534$ and $\beta \sim 0.627$ is the real root of $16 \beta^{3}-4 \beta^{2}+\beta-3=0$. We will prove Theorem 1.5, which states that there are constants $c_{2} \geqslant c_{1}>1$ such that $c_{1} \operatorname{ex}\left(n, C_{6}\right) \leqslant \operatorname{ex}^{*}\left(n, C_{6}\right) \leqslant c_{2} \operatorname{ex}\left(n, C_{6}\right)$. We will not attempt to optimize these constants. First we need a lemma. 
Lemma 3.1. Let $G$ be a bipartite graph on $n$ vertices with average degree $d=2 e(G) / n$. Suppose $G$ does not contain $K_{2, t}$ and has a proper edge-colouring with no rainbow $C_{6}$. Then $d<((11 t-12) n)^{1 / 3}+4$.

Proof of Lemma 3.1. We can assume that $G$ has minimum degree at least $d / 2$, as deleting a vertex of degree less than $d / 2$ does not decrease the average degree. As in the proof of Theorem 1.3 we see that the number of rainbow paths of length 3 satisfies

$$
R_{3} \geqslant \frac{1}{4} n d(d-1)(d-4)
$$

Given a pair of vertices $a, b$ we write $p_{a b}$ for the number of rainbow paths of length 3 that have endpoints $a$ and $b$. We claim that there are at least $\left(\begin{array}{c}p_{a b} \\ 2\end{array}\right)-(5 t-6) p_{a b}$ pairs of such paths $\left(a x y b, a x^{\prime} y^{\prime} b\right)$ for which $a x y b y^{\prime} x^{\prime}$ is a 6-cycle and $x^{\prime} y^{\prime}$ has the same colour as $x y$. To see this, fix any rainbow path $a x y b$. Since $G$ does not contain $K_{2, t}$ there are at most $t-2$ other paths of the form $a x y^{\prime} b$ and at most $t-2$ other paths of the form $a x^{\prime} y b$. This shows that there are at most $(t-2) p_{a b}$ (unordered) pairs $\left(a x y b, a x^{\prime} y^{\prime} b\right)$ for which $a x y b y^{\prime} x^{\prime}$ does not form a 6-cycle. Now consider $a x^{\prime} y^{\prime} b$ for which $a x y b y^{\prime} x^{\prime}$ is a 6-cycle. By assumption this is not rainbow so there are two edges with the same colour. There are at most 2 vertices $x^{\prime} \neq x$ such that $a x^{\prime}$ has a colour from the path $a x y b$, so at most $2(t-1)$ paths $a x^{\prime} y^{\prime} b$ where $a x^{\prime}$ has a colour from the path $a x y b$. Similarly there are at most $2(t-1)$ paths $a x^{\prime} y^{\prime} b$ where $y^{\prime} b$ has a colour from the path $a x y b$. Therefore there are at most $4(t-1) p_{a b}$ pairs $\left(a x y b, a x^{\prime} y^{\prime} b\right)$ such that $a x y b y^{\prime} x^{\prime}$ is a 6-cycle and one of $a x, y b$ has a colour from $a x^{\prime} y^{\prime} b$ or one of $a x^{\prime}, y^{\prime} b$ has a colour from $a x y b$. Since there is no rainbow $C_{6}$, for any 6-cycle axyby $y^{\prime} x^{\prime}$ not covered by the above exceptions the edges $x y$ and $x^{\prime} y^{\prime}$ have the same colour. It follows that there are at least $\left(\begin{array}{c}p_{a b} \\ 2\end{array}\right)-(t-2) p_{a b}-4(t-1) p_{a b}=\left(\begin{array}{c}p_{a b} \\ 2\end{array}\right)-(5 t-6) p_{a b}$ pairs $\left(a x y b, a x^{\prime} y^{\prime} b\right)$ for which $a x y b y^{\prime} x^{\prime}$ is a 6-cycle and $x^{\prime} y^{\prime}$ has the same colour as $x y$.

Call a path special if its first and last edges have the same colour and no other pair of edges have the same colour. (Note that this is slightly different to the definition used in the proof of Theorem 1.3.) A special path of length $k$ contains 2 rainbow paths of length $k-1$, and each rainbow path of length $k-1$ is contained in at most 2 special paths of length $k$, so the number of rainbow paths of length $k-1$ is an upper bound on the number of special paths of length $k$. To each pair $\left(a x y b, a x^{\prime} y^{\prime} b\right)$ for which $a x y b y^{\prime} x^{\prime}$ is a 6-cycle and $x^{\prime} y^{\prime}$ has the same colour as $x y$ we can associate the two special paths of length four $x y b y^{\prime} x^{\prime}$ and $y x a x^{\prime} y^{\prime}$. Also, each special path of length four belongs to at most $t-16$-cycles (as there is no $K_{2, t}$ ), and it is counted by exactly one partition of any such 6-cycle into two rainbow paths of length 3 . It follows that there are at least $\frac{2}{t-1} \sum_{a, b}\left(\left(\begin{array}{c}p_{a b} \\ 2\end{array}\right)-(5 t-6) p_{a b}\right)$ special paths of length 4 . As noted above, the number of special paths of length 4 is at most the number of rainbow paths of length 3 , which equals $\sum_{a, b} p_{a b}$ by definition. We conclude that

$$
\frac{2}{t-1} \sum_{a, b}\left(\left(\begin{array}{c}
p_{a b} \\
2
\end{array}\right)-(5 t-6) p_{a b}\right) \leqslant \sum_{a, b} p_{a b} .
$$


This may be re-written as $\sum_{a, b} p_{a b}^{2} \leqslant(11 t-12) \sum_{a, b} p_{a b}$. By the Cauchy-Schwarz inequality

$$
\sum_{a, b} p_{a b}^{2} \geqslant\left(\begin{array}{l}
n \\
2
\end{array}\right)^{-1}\left(\sum_{a, b} p_{a b}\right)^{2}
$$

and so we see that $\sum_{a, b} p_{a b} \leqslant(11 t-12)\left(\begin{array}{l}n \\ 2\end{array}\right)$. Recalling that $R_{3}=\sum_{a, b} p_{a b}$ and equation (3.2) we get $\frac{1}{2} n d(d-1)(d-4) \leqslant(11 t-12)\left(\begin{array}{l}n \\ 2\end{array}\right)$, which gives $d<(1+o(1))((11 t-12) n)^{1 / 3}+4$. This completes the proof.

Proof of Theorem 1.5. We start with the upper bound. Let $G$ be a graph on $n$ vertices that is properly coloured with no rainbow $C_{6}$. It contains a bipartite subgraph $G^{\prime}$ with $e\left(G^{\prime}\right) \geqslant e(G) / 2$. We say that a subgraph $K_{2, t}$ of $G^{\prime}$ is maximal if it is not contained in $K_{2, t^{\prime}}$ for any $t^{\prime}>2$. We claim that if $G^{\prime}$ contains a maximal $K_{2, s}$ and a maximal $K_{2, t}$ with $s, t \geqslant 9$ then they must be edge-disjoint.

For suppose that $\left(A_{1}, B_{1}\right)$ is a maximal $K_{2, s}$ with $\left|A_{1}\right|=2,\left|B_{1}\right|=s \geqslant 9,\left(A_{2}, B_{2}\right)$ is a maximal $K_{2, t}$ with $\left|A_{2}\right|=2,\left|B_{2}\right|=t \geqslant 9$, and $x y$ is a common edge. Consider first the case when $x \in A_{1} \cap A_{2}$ and $y \in B_{1} \cap B_{2}$. By maximality we have $A_{1}=\left\{x, z_{1}\right\}$ and $A_{2}=\left\{x, z_{2}\right\}$ with $z_{1} \neq z_{2}$. Let $c_{1}$ be the colour of $y z_{1}$ and $c_{2}$ of $y z_{2}$. There are at most 4 vertices $b_{1}$ in $B_{1}$ such that $x b_{1}$ or $z_{1} b_{1}$ has has colour $c_{1}$ or $c_{2}$, so we can choose $b_{1} \in B_{1}$ so that $x b_{1}$ has colour $c_{3}, z_{1} b_{1}$ has colour $c_{4}$ and $c_{1}, \ldots, c_{4}$ are all different. Now there are at most 8 vertices $b_{2}$ in $B_{2}$ so that $x b_{2}$ or $z_{2} b_{2}$ has a colour among $c_{1}, \ldots, c_{4}$. Choosing any other $b_{2}$ we obtain a rainbow 6-cycle $x b_{1} z_{1} y z_{2} b_{2}$. Now consider the case when $x \in A_{1} \cap B_{2}$ and $y \in B_{1} \cap A_{2}$. Write $A_{1}=\left\{x, z_{1}\right\}$ and $A_{2}=\left\{y, z_{2}\right\}$. Let $c_{1}$ be the colour of $y z_{1}$ and $c_{2}$ of $x z_{2}$. There are at most 4 vertices $b_{1}$ in $B_{1}$ such that $x b_{1}$ or $z_{1} b_{1}$ has has colour $c_{1}$ or $c_{2}$, so we can choose $b_{1} \in B_{1}$ so that $x b_{1}$ has colour $c_{3}, z_{1} b_{1}$ has colour $c_{4}$ and $c_{1}, \ldots, c_{4}$ are all different. Now there are at most 8 vertices $b_{2}$ in $B_{2}$ so that $y b_{2}$ or $z_{2} b_{2}$ has a colour among $c_{1}, \ldots, c_{4}$. Choosing any other $b_{2}$ we obtain a rainbow 6 -cycle $x b_{1} z_{1} y b_{2} z_{2}$. It follows that any edge of $G^{\prime}$ belongs to at most one maximal $K_{2, t}$ with $t \geqslant 9$.

Suppose that $(A, B)$ is a maximal $K_{2, t}$ with $A=\left\{a_{1}, a_{2}\right\}$ and $|B|=t \geqslant 9$. Delete from $G^{\prime}$ all edges joining $a_{1}$ to $B$. Repeat this process as long as there is any (maximal) $K_{2, t}$ with $t \geqslant 9$. Note that we have considered mutually disjoint sets of edges and deleted half of each, so we have deleted at most half of the edges of $G^{\prime}$. The remaining graph $G^{\prime \prime}$ contains no $K_{2,9}$. By Lemma 3.1 it has average degree $d^{\prime \prime}<(1+o(1))(87 n)^{1 / 3}$. Therefore $e(G) \leqslant 2 e\left(G^{\prime}\right) \leqslant 4 e\left(G^{\prime \prime}\right)=2 d^{\prime \prime} n<9 n^{4 / 3}$ for large $n$.

There is a lower bound $\operatorname{ex}^{*}\left(n, C_{6}\right) \geqslant \operatorname{ex}\left(n, C_{6}\right)=\Omega\left(n^{4 / 3}\right)$, but here we will give a better construction to show $\operatorname{ex}^{*}\left(n, C_{6}\right)>c \operatorname{ex}\left(n, C_{6}\right)$ with $c>1$. Suppose $n$ is even and consider a graph $G_{0}$ on $n / 2$ vertices with no cycle of length at most 6 . Let $G$ be the two-point blow-up of $G_{0}$, i.e., for each vertex $v \in G_{0}$ there are two vertices $v_{0}, v_{1}$ in $G$, and for each edge $u v \in G_{0}$ we have all four edges $u_{i} v_{j}, 0 \leqslant i, j \leqslant 1$ in $G$. Choose an arbitrary proper edge-colouring $c_{0}$ of $G_{0}$. We define an edge-colouring $c$ of $G$ by the rule $c\left(u_{i} v_{j}\right)=\left(c_{0}(u v), i+j \bmod 2\right)$. By this we mean that the colour of an edge is an ordered pair: the first element is the colour of the edge in $G_{0}$ it came from, and the second element is chosen to be 0 or 1 in a way that ensures that the resulting edge-colouring is proper. 
We claim that $G$ has no rainbow $C_{6}$. For suppose $C=a_{\alpha} b_{\beta} c_{\gamma} d_{\delta} e_{\epsilon} f_{\zeta}$ is a 6-cycle in $G$. Then abcdef is a closed walk in $G_{0}$, which has no cycle of length at most 6 , and it is easy to see that it must consist of a path of length 3 traversed in both directions. Without loss of generality $a$ and $d$ are the endpoints of this path. Then $e=c$ and $f=b$, so $\epsilon=\gamma+1$ and $\zeta=\beta+1$, which gives $\epsilon+\zeta=\gamma+\beta(\bmod 2)$. Thus the edges $b_{\beta} c_{\gamma}$ and $e_{\epsilon} f_{\zeta}$ have the same colour, so $C$ is not rainbow.

We can choose the graph $G_{0}$ to have $e\left(G_{0}\right)=(n / 4)^{4 / 3}+O(n)$ (see Remark (3) following this proof.) Then $\operatorname{ex}^{*}\left(n, C_{6}\right) \geqslant e(G)=4 e\left(G_{0}\right)=4^{-1 / 3} n^{4 / 3}+O(n) \geqslant(1+\lambda+o(1)) \operatorname{ex}\left(n, C_{6}\right)$, where, using the upper bound for $\operatorname{ex}\left(n, C_{6}\right)$ quoted at the beginning of this subsection, one may calculate that $\lambda \geqslant 4^{-1 / 3} \beta^{-1}-1>1 / 250$. This completes the proof of the theorem. $\square$

Remarks. (1) It follows from the proof that an edge-coloured bipartite graph on $2 n$ vertices with no rainbow $C_{6}$ has at most $\frac{1}{2} 9(2 n)^{4 / 3}<12 n^{4 / 3}$ edges (for large $n$ ). Applying this to a bipartite Cayley graph we see that in any abelian group of order $n$, a $B_{3}^{*}$-set can have at most $12 n^{1 / 3}$ elements.

(2) We have made no attempt to optimize the constants in our arguments, but it seems interesting that we have a purely combinatorial argument that gives the correct order of magnitude.

(3) The construction given in the above proof may be generalized as follows. Write $z(n, H)$ for the maximum number of edges in an $H$-free bipartite graph with $n$ vertices in each part, and let $z^{*}(n, H)$ denote the rainbow analogue of this definition. Applying the construction when $G_{0}$ is a bipartite graph with no cycles of length at most $2 k$ we see that $z^{*}\left(2 n, C_{2 k}\right) \geqslant 4 z\left(n, C_{4}, \ldots, C_{2 k}\right)$ for any $k$. It is shown in [17] that $z\left(n, C_{6}\right) \leqslant 2^{1 / 3} n^{4 / 3}+O(n)$, and there is a lower bound $z\left(n, C_{4}, C_{6}\right) \geqslant n^{4 / 3}+O(n)$ attributed to Benson [6]. Therefore $z^{*}\left(n, C_{6}\right) \geqslant 4 z\left(n / 2, C_{4}, C_{6}\right) \geqslant 4(n / 2)^{4 / 3}+O(n) \geqslant(1+o(1)) 2^{1 / 3} z\left(n, C_{6}\right)$.

\subsection{Excluding all cycles}

Now we will consider the problem of excluding any rainbow cycle. Note that the ordinary Turán problem is easy in this case: an acyclic graph on $n$ vertices has at most $n-1$ edges, with equality for a tree. On the other hand, we can construct graphs with order $n \log n$ edges that can be properly coloured with no rainbow cycle.

One construction is the $m$-cube, a bipartite graph in which the vertices are all subsets of $\{1, \ldots, m\}$ and for any $A \subset\{1, \ldots, m\}$ and $i \in A$ there is an edge between $A$ and $A \backslash\{i\}$ of colour $i$. There are no rainbow cycles in this graph, and in fact every cycle of length $2 k$ uses at most $k$ different colours. Indeed, if a cycle contains an edge $(A, A \backslash\{i\})$ of colour $i$ then the path continuing along the cycle from $A \backslash\{i\}$ must again use at least one edge of colour $i$ in order to reach $A$, which contains the element $i$. The $m$-cube has $n=2^{m}$ vertices and $m 2^{m-1}=\frac{1}{2} n \log _{2} n$ edges. 
An improvement in the constant can be obtained by using as a building block the bipartite graph $K_{3,3}$ with parts $\left\{x_{0}, x_{1}, x_{2}\right\}$ and $\left\{y_{0}, y_{1}, y_{2}\right\}$ in which the edge $x_{i} y_{j}$ has colour $i-j \bmod 3$. Clearly this contains no rainbow cycles.

Proof of Proposition 1.6. We construct a graph whose vertices are sequences of length $m$ in which each term is one of $x_{0}, x_{1}, x_{2}, y_{0}, y_{1}, y_{2}$ (notation as above). Two sequences $z=\left(z_{1}, \ldots, z_{m}\right)$ and $z^{\prime}=\left(z_{1}^{\prime}, \ldots, z_{m}^{\prime}\right)$ are adjacent if there is some $s$ such that $z_{t}=z_{t}^{\prime}$ for $t \neq s$ and $z_{s} z_{s}^{\prime}$ is an edge of the building block $K_{3,3}$. We colour such an edge $z z^{\prime}$ with the pair $(s, c)$, where $c$ is the colour of $z_{s} z_{s}^{\prime}$. Consider any cycle $z^{1}, \ldots, z^{k}$. For any $1 \leqslant s \leqslant m$ the terms $z_{s}^{1}, \ldots, z_{s}^{k}, z_{s}^{1}$ form a sequence of vertices in $K_{3,3}$ in which each term is either adjacent or equal to the one preceding it. There is at least one $s$ for which these terms are not all equal. Then there is a closed walk in $K_{3,3}$ whose edges appear as adjacent members of $z_{s}^{1}, \ldots, z_{s}^{k}$, so some colour is repeated. It follows that there are no rainbow cycles. This graph has $n=6^{m}$ vertices and $6^{m} \cdot 3 m / 2=\frac{3}{2 \log _{2} 6} n \log _{2} n>0.58 n \log _{2} n$ edges.

We do not have a good upper bound for the problem of finding a rainbow cycle, but we can determine the order of magnitude for finding a cycle with more than half as many colours as edges.

Proof of Theorem 1.7. A construction with $\frac{1}{2} n \log _{2} n$ edges when $n$ is a power of 2 was described above. For the upper bound let $G$ be a graph on $n$ vertices so that any cycle of length $2 k$ uses at most $k$ different colours for any $k$. Let $d=2 e(G) / n$ be the average degree. By deleting vertices of small degree we can assume that the minimum degree is at least $d / 2$. We claim that the number of rainbow paths of length $k$ satisfies

$$
R_{k}>2 n \prod_{i=0}^{k-1}(d / 2-i) \text {. }
$$

The proof is a slight improvement on that given for equation (3.1) in Theorem 1.3. As before we have $R_{2} \geqslant n\left(\begin{array}{l}d \\ 2\end{array}\right)$, which is larger than $2 n(d / 2)(d / 2-1)$. For $t \geqslant 2$ each rainbow path of length $t+1$ contains 2 rainbow paths of length $t$. Also, given a rainbow path of length $t$, each of its endpoints is incident to at least $d / 2$ edges, of which only one has an endpoint on the path (otherwise there would be a rainbow cycle) and at most $t-1$ others have a colour that appears on the path, so it can be extended to a rainbow path of length $t+1$ in at least $2(d / 2-t)$ ways. Therefore $R_{t+1} \geqslant(d / 2-t) R_{t}$, which proves the claim.

Note that if a pair of vertices is joined by two rainbow paths then they have the same length. For the symmetric difference of the paths is a disjoint union of cycles, and if one of them is longer then it will contribute more than half of the edges of one of these cycles. Since the path is rainbow this cycle will have more than half as many colours as edges, which is a contradiction. The same argument shows that in fact the two paths use exactly the same set of colours.

For each $k$ let $H_{k}$ be the graph consisting of all pairs $a b$ for which there is a rainbow path of length $k$ from $a$ to $b$ in $G$. Consider such a path using colours $c_{1}, \ldots, c_{k}$. We showed above that any other such path uses a permutation of these colours. Since $G$ is 
properly coloured it is uniquely determined by the permutation, so there are at most $k$ ! such paths. Therefore $e\left(H_{k}\right) \geqslant R_{k} / k$ !, which gives $\sum_{k \geqslant 1} R_{k} / k ! \leqslant \sum_{k \geqslant 1} e\left(H_{k}\right) \leqslant\left(\begin{array}{l}n \\ 2\end{array}\right)$, since for $k \neq k^{\prime}$ the graphs $H_{k}$ and $H_{k^{\prime}}$ are edge-disjoint. Recalling that $R_{k}>2 n \prod_{i=0}^{k-1}(d / 2-i)$, we see that

$$
2 n\left(2^{d / 2}-1\right)=2 n \sum_{k \geqslant 1}\left(\begin{array}{c}
d / 2 \\
k
\end{array}\right)<\sum_{k \geqslant 1} R_{k} / k ! \leqslant\left(\begin{array}{l}
n \\
2
\end{array}\right),
$$

which gives $d<2 \log _{2}(n+3)-4$, i.e., $e(G)<n \log _{2}(n+3)-2 n$.

Remark. A properly edge-coloured $d$-regular graph on $n$ vertices has at least $n d(d-$ 1) $\cdots(d-k+1)=n k !\left(\begin{array}{l}d \\ k\end{array}\right)$ rainbow walks of length $k$. It is natural to conjecture that a graph with average degree $d$ should have at least this many rainbow walks of length $k$. Under the assumptions of the above theorem all rainbow walks are in fact paths, so if this conjecture is true we would have $R_{k} \geqslant \frac{1}{2} n k !\left(\begin{array}{l}d \\ k\end{array}\right)$ and so $\left(\begin{array}{l}n \\ 2\end{array}\right) \geqslant \sum_{k \geqslant 1} R_{k} / k ! \geqslant \frac{1}{2} n \sum_{k \geqslant 1}\left(\begin{array}{l}d \\ k\end{array}\right)=n\left(2^{d}-1\right)$, i.e., $d \leqslant \log _{2} n$. This would show that the lower bound is tight, not just asymptotically but exactly when $n$ is a power of 2 .

We conclude this subsection with an argument very similar to the previous proof that gives a girth result for this weaker condition on cycle colourings, under a weaker assumption than the type used in Theorem 1.3.

Theorem 3.2. Suppose $k>1$ and let $G$ be a graph on $n$ vertices so that any cycle of length $2 t$ uses at most $t$ different colours for any $t \leqslant k$. Then $e(G)<(1+o(1))(k !)^{1 / k} n^{1+1 / k}$.

Proof of Theorem 3.2. Let $G$ be a graph on $n$ vertices so that any cycle of length $2 t$ uses at most $t$ different colours for any $t \leqslant k$. Let $d=2 e(G) / n$ be the average degree. By deleting vertices of small degree we can assume that the minimum degree is at least $d / 2$. As in the previous proof we have $R_{k}>2 n \prod_{i=0}^{k-1}(d / 2-i)$. Defining $H_{k}$ as before we have $R_{k} / k ! \leqslant e\left(H_{k}\right) \leqslant\left(\begin{array}{l}n \\ 2\end{array}\right)$. Therefore $d<(1+o(1)) 2(k ! n / 4)^{1 / k}$, so $e(G)<(1+o(1)) k !^{1 / k} n^{1+1 / k}$.

\section{Concluding remarks and open problems}

(1) There is a natural extension of our problem to hypergraphs: if $\mathscr{F}$ is a fixed $r$-uniform hypergraph then $\operatorname{ex}(n, \mathscr{F})$ denotes the number of edges in the largest $\mathscr{F}$-free $r$-uniform hypergraph on $n$ vertices, and $\operatorname{ex}^{*}(n, \mathscr{F})$ is the maximum number of edges in a properly edge-coloured $r$-uniform hypergraph on $n$ vertices with no rainbow $\mathscr{F}$. We remark that the arguments of Proposition 1.1 can be extended to show that $\operatorname{ex}^{*}(n, \mathscr{F}) \leqslant \operatorname{ex}(n, \mathscr{F})+o\left(n^{r}\right)$. The details are very similar, and instead of the Erdős-Stone theorem one uses ErdősSimonovits supersaturation [13], which states that $\operatorname{ex}(n, \mathscr{F}(t)) \leqslant \operatorname{ex}(n, \mathscr{F})+o\left(n^{r}\right)$. (Here $\mathscr{F}(t)$ denotes the $t$-point blow-up of $\mathscr{F}$.) Since even the ordinary Turán theory of hypergraphs is poorly understood, we will not study this question any further here. 
(2) We have seen that for non-bipartite $H$ the rainbow Turán number $\operatorname{ex}^{*}(n, H)$ is asymptotically equal to the ordinary Turán number ex $(n, H)$. For bipartite graphs we have seen some evidence that these quantities may have the same order of magnitude. They are not asymptotically equal in general, as we saw with the examples of a path of length 3 and a six-cycle. It seems plausible that other bipartite graphs, such as even cycles and complete bipartite graphs, should also exhibit this phenomenon. Perhaps it will be helpful for intuition in rainbow Turán problems to prove some natural structural properties. For example, is it true that a properly edge-coloured graph $G$ with no rainbow $H$ has a proper edge-colouring using the minimum possible number of colours (i.e., $\chi^{\prime}(G)$ ) which also has no rainbow $H$ ?

(3) In Section 3.3, we observed that an $n$-vertex properly edge-coloured $d$-regular graph contains $n k !\left(\begin{array}{l}d \\ k\end{array}\right)$ rainbow walks of length $k$, and conjecture that this is a lower bound for the number of rainbow walks of length $k$ in any properly coloured $n$ vertex graph of average degree $d$. For some intuition as to why this conjecture might be true, we cite an inequality of Blakley and Roy [7] that implies that a graph with average degree $d$ has at least $n d^{k}$ walks of length $k$. Secondly, there is the following result of Alon, Hoory and Linial that may be found within the proof in [1]. Consider a walk of length $k$ using the edges $e_{1}, \ldots, e_{k}$ in succession. It is a non-returning walk if we never have $e_{i}=e_{i+1}$. It is shown in [1] that a graph with $n$ vertices and average degree $d$ has at least $n d(d-1)^{k-1}$ non-returning walks of length $k$. A possible generalization of these results is to count walks of length $k$ in which there are $a_{i}$ forbidden edges at the ith step (possibly depending on the walk so far). One might think that a graph with $n$ vertices and average degree $d$ has at least $n \prod_{i=1}^{k}\left(d-a_{i}\right)$ such walks. This would include our conjecture on rainbow walks as a special case.

(4) We have mentioned a number of open problems throughout the paper, but for the convenience of the reader we conclude by repeating the two we consider most important.

(i) How many edges can a properly edge-coloured graph on $n$ vertices have if it contains no rainbow cycles?

(ii) How many edges can a properly edge-coloured graph on $n$ vertices have if it contains no rainbow $C_{2 k}$ ?

\section{Acknowledgement}

We thank an anonymous referee for suggestions that improved the presentation of this paper, and for drawing certain references to our attention, including the construction of Maamoun and Meyniel used in Section 2.

\section{References}

[1] Alon, N., Hoory, S. and Linial, N. (2002) The Moore bound for irregular graphs. Graphs Combin. 18 53-57.

[2] Alon, N., Jiang, T., Miller, Z. and Pritikin, D. (2003) Properly coloured subgraphs and rainbow subgraphs in edge-colourings with local constraints. Random Struct. Alg. 23 409-433. 
[3] Alon, N., Krivelevich, M. and Sudakov, B. (2003) Turán numbers of bipartite graphs and related Ramsey-type questions. Combin. Probab. Comput. 12 477-494.

[4] Alon, N., Rónyai, L. and Szabó, T. (1999) Norm-graphs: Variations and applications. J. Combin. Theory Ser. B 76 280-290.

[5] Behrend, F. A. (1946) On sets of integers which contain no three terms in arithmetical progression. Proc. Nat. Acad. Sci. USA 32 331-332.

[6] Benson, C. T. (1966) Minimal regular graphs of girths eight and twelve. Canad. J. Math. 18 1091-1094.

[7] Blakley, G. R. and Roy, P. (1965) A Hölder type inequality for symmetric matrices with nonnegative entries. Proc. Amer. Math. Soc. 16 1244-1245.

[8] Bondy, J. A. and Simonovits, M. (1974) Cycles of even length in graphs. J. Combin. Theory Ser. B 16 97-105.

[9] Bose, R. C. and Chowla, S. (1962/1963) Theorems in the additive theory of numbers. Comment. Math. Helv. 37 141-147.

[10] Chen, G., Schelp, R. and Wei, B. (2001) Monochromatic-rainbow Ramsey numbers. 14th Cumberland Conference Abstracts. http://www.msci.memphis.edu/balistep/Abstracts.html

[11] Erdős, P., Füredi, Z., Gould, R. J. and Gunderson, D. S. (1995) Extremal graphs for intersecting triangles. J. Combin. Theory Ser. B 64 89-100.

[12] Erdős, P. and Rado, R. (1950) A combinatorial theorem. J. London Math. Soc. 25 249-255.

[13] Erdős, P. and Simonovits, M. (1983) Supersaturated graphs and hypergraphs. Combinatorica 3 181-192.

[14] Erdős, P. and Stone, A. H. (1946) On the structure of linear graphs. Bull. Amer. Math. Soc. 52 1087-1091.

[15] Füredi, Z. (1996) An upper bound on Zarankiewicz' problem. Combin. Probab. Comput. 5 $29-33$.

[16] Füredi, Z. (1996) New asymptotics for bipartite Turán numbers. J. Combin. Theory Ser. A 75 $141-144$.

[17] Füredi, Z., Naor, A. and Verstraëte, J. (2006) On the Turán number for the hexagon. Adv. Math. 203 476-496.

[18] Graham, S. W. (1996) $B_{h}$ sequences. In Analytic Number Theory, Vol. 1 (Allerton Park, IL, 1995), Vol. 138 of Progr. Math., Birkhäuser, Boston, MA, pp. 431-449.

[19] Jamison, R. E., Jiang, T. and Ling, A. C. H. (2003) Constrained Ramsey numbers of graphs. J. Graph Theory 42 1-16.

[20] Kollár, J., Rónyai, L. and Szabó, T. (1996) Norm-graphs and bipartite Turán numbers. Combinatorica 16 399-406.

[21] Kővári, T., Sós, V. and Turán, P. (1954) On a problem of K. Zarankiewicz. Colloquium Math. 3 $50-57$.

[22] Lazebnik, F., Ustimenko, V. A. and Woldar, A. J. (1995) A new series of dense graphs of high girth. Bull. Amer. Math. Soc. 32 73-79.

[23] van Lint, J. H. and Wilson, R. M. (2001) A Course in Combinatorics, 2nd edn, Cambridge University Press.

[24] Maamoun, M. and Meyniel, H. (1984) On a problem of G. Hahn about coloured Hamiltonian paths in $K_{2 n}$. Discrete Math. 51 213-214.

[25] Moon, J. W. and Moser, L. (1962) On a problem of Turán. Magyar Tud. Akad. Mat. Kutat Int. Kzl. $7283-286$.

[26] Rödl, V. and Tuza, Zs. (1992) Rainbow subgraphs in properly edge-coloured graphs. Random Struct. Alg. 3 175-182.

[27] Roth, K. F. (1953) On certain sets of integers. J. London Math. Soc. 28 104-109.

[28] Ruzsa, I. Z. (1993) Solving a linear equation in a set of integers I. Acta Arith. 65 259-282. 
[29] Ruzsa, I. Z. and Szemerédi, E. (1978) Triple systems with no six points carrying three triangles. In Combinatorics: Proc. Fifth Hungarian Colloq., Keszthely, 1976, Vol. II, Colloq. Math. Soc. János Bolyai, Vol. 18, North-Holland, Amsterdam/New York, pp. 939-945,

[30] Simonovits, M. (1968) A method for solving extremal problems in graph theory, stability problems. In Theory of Graphs: Proc. Colloq. Tihany, 1966, Academic Press, New York, and Akad. Kiadó, Budapest, pp. 279-319.

[31] Singer, J. (1938) A theorem in finite projective geometry and some applications to number theory. Trans. Amer. Math. Soc. 43 377-385.

[32] Turán, P. (1954) On the theory of graphs. Colloquium Math. 3 19-30.

[33] Verstraëte, J. (2000) On arithmetic progressions of cycle lengths in graphs. Combin. Probab. Comput. 9 369-373. 